\title{
Nine Steps to Discover New Medicines from Traditional Sources: The Example of Persian Medicine
}

\author{
Seyyed Ali Mozaffarpur, MD, PhD, ${ }^{1}$ Mohsen Naseri, MD, PhD, ${ }^{2}$ Mohammad Kamalinejad, MSc, ${ }^{3}$ \\ Mohammad Ali Shams, PhD, ${ }^{4}$ Zahra Memariani, PharmD, PhD, ${ }^{5,6}$ Reyhaneh Moeini, MD, PhD, ${ }^{6}$ \\ Narjes Gorji, MD, PhD, ${ }^{6}$ Akram Moradi Farahani, MD, PhD, and Hoda Shirafkan, $\mathrm{PhD}^{7}$
}

\begin{abstract}
Objectives: Developing a new medicine is a costly and time-consuming process. This study presents a novel method to collate a new materia medica from previously documented traditional Persian medicine (TPM) references. A well-documented materia medica will facilitate a quick and easy process to find traditional medicine for specific symptoms or diseases.

Materials and methods: A nine-step algorithm is proposed to collate a new materia medica by searching and matching descriptions and references stated in various traditional Persian literature: (1) finding the equivalent of traditional description of symptoms or diseases; (2) determining the keywords to search in the references of TPM, including The Canon of Medicine (Book by Avicenna); (3) determining the best references of TPM for the aim of the study; (4) weighing up the references (a) based on the prominence of the author's prominence (b) determining whether the references are the author's own clinical findings or has citation from other scholars been used, (c) the popularity of the book that the references feature in, among other later scholars of TPM, the geographical and historical span across which the book has been distributed; (5) searching the references using keywords of symptoms or diseases; (6) finding the synonyms of terminologies in the various materia medicas and categorizing the findings; (7) revision (of references found) to ensure that nothing is neglected; (8) scoring the findings based on the defined criteria; (9) prioritization: sorting the materia medica based on the total scores. Some important economic, cultural or historical factors cannot be included in the main process of prioritization during the initial searching of the TPM materia medica. Therefore, it is necessary to take these factors into account after discovering the medicine and before using in practice to ascertain that they are a good match of a specific treatment, disease or symptom.
\end{abstract}

Conclusion: A process to compile a new materia medica has been proposed which will enable the quick and easy selection of herbal treatments for various signs and symptoms of illnesses.

Keywords: drug discovery, herbal, medicine, traditional, complementary therapies

\footnotetext{
${ }^{1}$ Traditional Medicine and History of Medical Sciences Research Center, Health Research Institute, Babol University of Medical Sciences, Babol, Iran.

${ }^{2}$ Traditional Clinical Trial Research Center, Shahed University, Tehran, Iran.

${ }^{3}$ School of Pharmacy, Shahid Beheshti University of Medical Sciences, Tehran, Iran.

${ }^{4}$ Department of English, Babol University of Medical Sciences, Babol, Iran.

${ }^{5}$ Department of History of Medical Sciences, School of Persian Medicine, Babol University of Medical Sciences, Babol, Iran.

${ }^{6}$ Department of Persian Medicine, School of Persian Medicine, Babol University of Medical Sciences, Babol, Iran.

${ }^{7}$ Social Determinants of Health Research Center, Health Research Institute, Babol University of Medical Sciences, Babol, Iran.
} 


\section{Introduction}

TnVenting nOvel DRUGs for old diseases is becoming increasingly important. ${ }^{1}$ Seeking new approaches to document medicinal plants and herbs will reduce the cost and the time required to process the medicine. ${ }^{2}$ This approach has been promoted in different fields of medicine and in different areas of the world. ${ }^{3-5}$ It is shown that $40 \%$ of the approved drugs include natural compounds or are inspired by them. ${ }^{6,7}$

A new and systematically compiled traditional medicine references, including ancient books or folk medicine, can help avoid the long time frames currently required to manufacture and test new affective herbal medicine. ${ }^{2}$ In some traditional medicine cultures such as Traditional Chinese Medicine, a regulatory system for new drug evaluation and registration in accordance with international standards has been developed ${ }^{8}$; however, the process of choosing the best herbal medicine for a particular symptom or disease has not been precisely developed. ${ }^{9}$

Traditional Persian medicine (TPM) is one of the oldest systems of medicine. ${ }^{10}$ There are $\sim 14,000$ nonrecurrent references, most of which are written in the Arabic or Persian languages. There is now a revival within traditional medicine, whereby, introducing new medications based on older TPM references are being developed. ${ }^{11-15}$ Most of these studies do not have any definite search strategies to select the best materia medica in the TPM references. 9

The authors aim to develop a theory of an algorithm to compile a new materia medica of herbal medicine from older and ancient literature for specific diseases or symptoms based on TPM references to facilitate an easier and less time-consuming process of selection of medicine.

\section{Procedure}

The procedure can be carried out in nine steps:

(1) Finding the traditional equivalent: To find a medication for any specific case (symptoms, signs, or diseases), it is necessary to find its approximate equivalent in TPM. It should be mentioned that symptoms and diseases in traditional and current medicine do not always necessarily match. Therefore, finding a perfect equivalent for every symptom and disease and establishing a one-to-one symmetry between currently used terminologies and the more archaic terminologies used in TPM. It is difficult, but should be done as the first step.

(2) Determining the keywords: Referring to traditional books on treatment and traditional materia medicas to find traditional keywords related to the symptom or disease under study. These keywords can be used to search in the references related to the topic. At this stage, even the keywords that are tentatively associated with the issue must be considered. The authors acknowledge that separating the explicit and implicit (referring to a mechanism relating to the authors' topic) keywords is a difficult process. Therefore, to be on the safe side, it is better to collect all the relevant keywords reported in the research.

(3) Determining the references: To find the best body of references, the following points should be taken into consideration: (a) The books found in materia medicas (a major kind of references in TPM, similar to books on pharmacology in modern medicine (are the best references.

(b) To assess the historical span for the use of a certain medication, it is better to refer to books from different geographical areas.

(c) It is recommended to use effective and popular books that establish their own style.

(d) At least 6-8 references will be required per disease or symptom.

(4) Weighing up of references: As TPM was developing through time, some TPM books have proved to be popular as a guide in helping with treatments. It is recommended that expert opinions be used in determining the references and weighing them. Alternatively, if a researcher does not have access to experts, it is better that all references be given the same weight.

(5) Searching among references: As herbal medicine in TPM is categorized to single ingredient or compound (more than one ingredient) medicine, this stage recommends to focus on herbal medicine that has singular ingredients for symptoms or diseases and does not recommend searching among combination medicines. It is important to know that texts on therapeutic effect of a herbal medicine in TPM references are declared in two ways: mostly the effect is written explicitly, but in some cases it is focused on a mechanism that an expert can find that it can be effective in a specific symptom of disease (the authors called it implicit). It is better to mark the implicit nature of such keywords when presenting the results of the study. Digitalization of the references can help doing this step faster and more precisely.

(6) Finding the synonyms and categorizing: As some materia medica might have had various names in different time periods and across different regions, the synonyms of the common keywords must be found in references on materia medica. It is suggested that the keyword and its synonyms are placed in a single column to facilitate referrals to the relevant references at a later date in a phrase table. Lastly, all the synonyms must be alphabetized in a single list.

(7) Revision: Since it is possible that a keyword is only implicitly mentioned (whereas explicitly discussed in other references) and could be overlooked in the first review. Therefore, when a keyword is found in a single reference, a new search must be launched to check all the previously investigated references to ensure that nothing is neglected. This is a crucial step as many of the implicit keywords affecting the scoring process are found in this stage. All the findings of this stage must be imported into the phrase table to be later evaluated and scored.

(8) Final scoring: After completion of the aforementioned seven steps, the table will be ready for scoring. The efficacy of a materia medica might be mentioned in various forms: (a) implicit reference to its therapeutic effect, (b) explicit reference to its therapeutic effect, (c) reference with different degrees of emphasis expressed by using a range of evaluative phrases such as "is useful," "has an observable ef- 
fect," “is greatly influential," “is unique," "has a wonderful effect," and "is extremely useful.",

It is recommended to rate every medication on a scale ranging from -3 to +3 . The scores reflect the degree of emphasis associated with the effect of materia medica. Sometimes in addition to the author's opinion, references present pros and cons proposed by other scholars. In such cases, if the other authors are not already included in the authors' list of references, it is recommended to rate them as well (within the -3 to +3 scale). After scoring each phrase or sentence and multiplying it by the weight of the reference, the sum of all the produced numbers for every materia medica in all the relevant references is calculated; the score of a reference excluding the target keyword is 0 . In the end, the total score of each materia medica in every row of the table must be calculated and presented in the last column.

(9) Prioritization: After calculating the total scores, the materia medica should be sorted based on their value. Figure 1 shows all steps in a schematic algorithm.

Since this prioritization is merely based on the effectiveness of the materia medica (regardless of economic or cultural factors), if clinical work is required to produce the materia medica, it is necessary to include the other factors into the process of prioritization. These factors include the following:

Consensus on its nature: There are controversies on the nature of some materia medica and matching them to the new botanical categorizations. Therefore, those whose identity is a matter discussion hence cannot be definitely

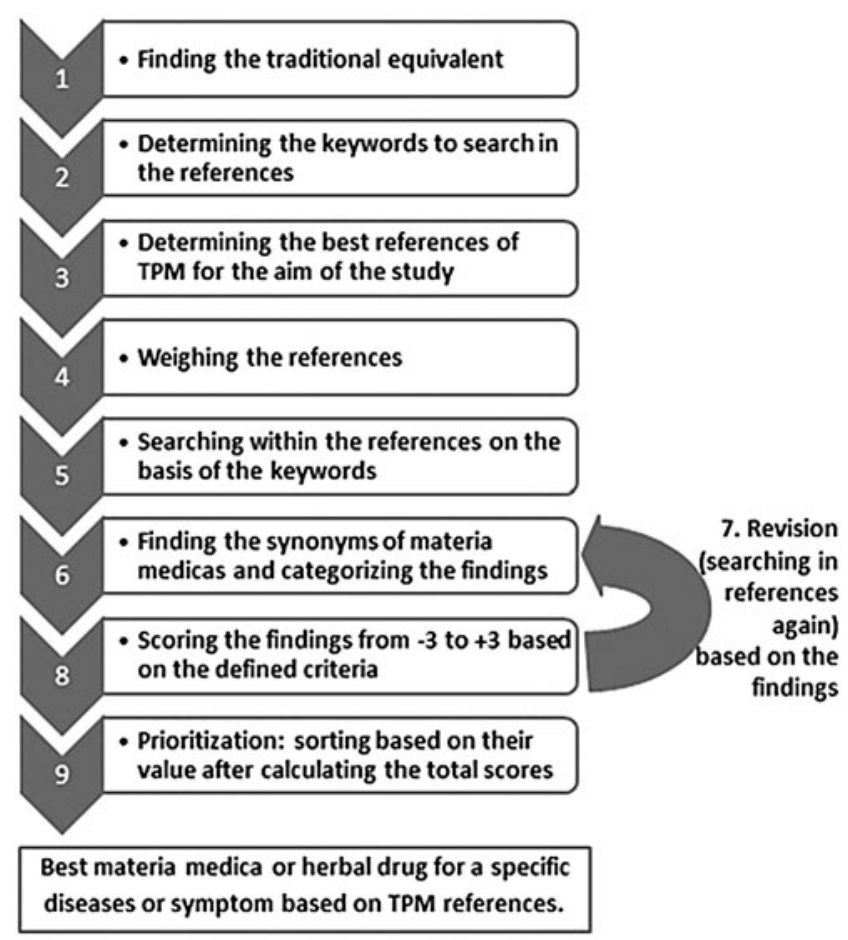

FIG. 1. Schematic algorithm to achieve the best new materia medica or herbal drug for a specific diseases or symptom based on traditional Persian medicine (TPM) references. categorized under modern botanical division have lower priority compared with the ones that do not arouse such controversy.

Experience of healers or physicians: Every materia medica whose experience is on it at present can be better judged.

Accessibility: Some materia medica may be very effective but not easily accessible (e.g., mummy). Logically, such items are not a priority.

Cost-effectiveness: Being cost-effective is considered an important advantage for a medication in Persian medicine. Food-likeness: Food-likeness ensures more tolerance and duration of medication adherence.

Lower dosage and less side effects: The closer a drug is to the body's natural equilibrium, the less side effects it has. Therefore, the tolerance will be higher.

Route of application: Given the higher potential of using topical medication in TPM, as side effects usually result from the use of oral medication. Finding topical medications to replace oral medications is a priority (e.g., rubbing Mostaka oil (Mastic oil) on the stomach is useful in treating some digestive conditions).

Ease of use: Medicines that are easier to use (e.g., pills and syrups) enjoy more public acceptance.

Good taste: Tasty medications are taken up much better; moreover, they are more effective because the patient is using them with enthusiasm.

Environmentally friendly: Of materia medicas having almost the same effect, the one less harmful to nature is definitely preferable.

Ease of preparation: Some medications might be effective but need a complex or expensive production process; this is a disadvantage and reduces their priority.

Durability: More durable herbal drugs are favored both by the patients and the drug suppliers.

\section{Acknowledgments}

The authors extend their profound gratitude to all the respected scholars who reviewed this article and suggested useful modifications, especially Dr. Mohammad Mahdi Isfahani. They also thank Ms. Yasamin Veziari for editing the article.

\section{Author Disclosure Statement}

No competing financial interests exist.

\section{Funding Information}

No funding was received for this article.

\section{References}

1. Gardner CR, Walsh CT, Almarsson Ö. Drugs as materials: Valuing physical form in drug discovery. Nat Rev Drug Discov 2004;3:926.

2. Lone PA, Bhardwaj AK, Bahar FA. Study of indigenous/ traditional medicinal plant knowledge: An endeavour towards new drug discovery. AJTCAM 2015;12:73-95.

3. Russo P, Frustaci A, Del Bufalo A, et al. From traditional European medicine to discovery of new drug candidates for the treatment of dementia and Alzheimer's disease: Acetylcholinesterase inhibitors. Curr Med Chem 2013;20: 976-983. 
4. Divino da Rocha M, Pereira Dias Viegas F, Cristina Campos $\mathrm{H}$, et al. The role of natural products in the discovery of new drug candidates for the treatment of neurodegenerative disorders II: Alzheimer's disease. CNS Neurol Disord Drug Targets 2011;10:251-270.

5. Dereli FTG, Ilhan M, Akkol EK. New drug discovery from medicinal plants and phytoconstituents for depressive disorders. CNS Neurol Disord Drug Targets 2019;18:92-102.

6. Seca A, Pinto D. Plant secondary metabolites as anticancer agents: Successes in clinical trials and therapeutic application. Int J Mol Sci 2018;19:263.

7. Majolo F, Delwing LKdOB, Marmitt DJ, et al. Medicinal plants and bioactive natural compounds for cancer treatment: Important advances for drug discovery. Phytochem Lett 2019;31:196-207.

8. Wan-Ying W, Jin-Jun H, Hua-Li L, et al. TCM-based new drug discovery and development in China. CJNM 2014;12: 241-250.

9. Mozaffarpur SA, Khodadust M, Shirafkan H, et al. Introducing a model for prioritization of drugs, based on Iranian traditional medicine references [in Persian]. Med Hist J 2014;6:11-28.

10. Persian medicine. Lancet 1904;163:P1584-P1585.

11. Memariani Z, Moeini R, Hamedi SS, et al. Medicinal plants with antithrombotic property in Persian medicine: A mechanistic review. J Thromb Thrombolys 2018;45: $158-179$.
12. Ardakani Movaghati MR, Yousefi M, Saghebi SA, et al. Efficacy of black seed (Nigella sativa L.) on kidney stone dissolution: A randomized, double-blind, placebo-controlled, clinical trial. Phytotherapy Research 2019;33:1404-1412.

13. Hosseini SMa-R, Zibaee S, Yousefi M, et al. Camel milk with pegylated interferon Alfa-2a and Ribavirin for treatmentnaive chronic hepatitis $C$ genotype 2/3: An open-label, randomized controlled trial. IRCMJ 2017;19, DOI: 10.5812/ ircmj.13529.

14. Vahid H, Bonakdaran S, Khorasani ZM, et al. Effect of Capparis spinosa extract on metabolic parameters in patients with type-2 diabetes: A randomized controlled trial. Endocr Metab Immune 2019;19:100-107.

15. Mahjoub F, Salari R, Yousefi M, et al. Effect of Pistacia atlantica kurdica gum on diabetic gastroparesis symptoms: A randomized, triple-blind placebo-controlled clinical trial. Electronic Physician 2018;10:6997.

Address correspondence to: Hoda Shirafkan, PhD Health Research Institute Babol University of Medical Sciences Ganjafrooz Street, Babol Mazandaran, Iran 47176-47745

E-mail: hodashirafkan@gmail.com 